Draft version October 1, 2018

Preprint typeset using LTEX style emulateapj v. 5/2/11

\title{
THE LOST DWARFS OF CENTAURUS A AND THE FORMATION OF ITS DARK GLOBULAR CLUSTERS
}

\author{
Mia Sauda Bovill ${ }^{1,2}$, Thomas H. Puzia ${ }^{2}$, Massimo Ricotti $^{3}$, Matthew A. Taylor ${ }^{2,4}$ \\ 1 - Space Telescope Science Institute, 3700 San Martin Drive, Baltimore, MD 21218, USA \\ 2 - Institute of Astrophysics, Pontificia Universidad Católica de Chile, Av. Vicuña Mackenna 4860, 7820436 Macul, Santiago, Chile \\ 3 - Department of Astronomy, University of Maryland, College Park, MD 20740, USA and \\ 4 - European Southern Observatory, Alonso de Corboda 3107, Vitacura, Santiago, Chile \\ Draft version October 1, 2018
}

\begin{abstract}
We present theoretical constraints for the formation of the newly discovered dark star clusters (DSCs) with high mass-to-light $(\mathcal{M} / \mathcal{L})$ ratios, from Taylor et al $(2015)$. These compact stellar systems photometrically resemble globular clusters (GCs) but have dynamical $\mathcal{M} / \mathcal{L}$ ratios of $\sim 10-100$, closer to the expectations for dwarf galaxies. The baryonic properties of the dark star clusters (DSCs) suggest their host dark matter halos likely virialized at high redshift with $\mathcal{M}>10^{8} M_{\odot}$. We use a new set of high-resolution N-body simulations of Centaurus A to determine if there is a set of $z=0$ subhalos whose properties are in line with these observations. While we find such a set of subhalos, when we extrapolate the dark matter density profiles into the inner $20 \mathrm{pc}$, no dark matter halo associated with Centaurus A in our simulations, at any redshift, can replicate the extremely high central mass densities of the DSCs. Among the most likely options for explaining $10^{5}-10^{7} M_{\odot}$ within $10 \mathrm{pc}$ diameter subhalos is the presence of a central massive black hole. We, therefore, propose that the DSCs are remnant cusps of stellar systems surrounding the central black holes of dwarf galaxies which have been almost completely destroyed by interactions with Centaurus A.
\end{abstract}

\section{INTRODUCTION}

The formation of compact stellar systems (CSSs), such as globular clusters (GCs), ultra-compact dwarfs (UCDs), and dwarf-globular transition objects (DGTOs) is a major contemporary problem for modern numerical simulations (e.g. Renaud et al. 2013, 2015, and references therein). Recent studies have revealed surprisingly complex properties, such as central black holes (BHs) and extended star-formation histories (e.g. Seth et al. 2014, Norris et al. 2015).

Recently, Taylor et al. (2015) (hereafter T15) derived dynamical masses for 112 of the brightest CSSs in the nearby giant elliptical galaxy NGC 5128, (Centaurus A; $D=3.8 \pm$ $0.1 \mathrm{Mpc}$, Harris et al. 2010). In addition to the expected GCs and UCDs, they found evidence for so-called dark star clusters (DSCs) which exhibit disproportionately high mass-tolight $(\mathcal{M} / \mathcal{L})$ ratios but have typical Local Group GC luminosities and structural properties. In comparison to UCDs, which show a scaling relation as $\mathcal{M}_{\text {dyn }} / \mathcal{L} \propto \mathcal{M}_{\text {dyn }}^{0.33 \pm 0.04}$, the DSC sequence exhibits a significantly steeper scaling, i.e. $\propto \mathcal{M}_{\mathrm{dyn}}^{0.79 \pm 0.04}$. No peculiarities in the spatial distribution of these DSCs compared to UCDs or the normal GC population were found. The possible origins of DSCs are presently unclear. Excluding observational biases (see Taylor et al. 2015 for a detailed discussion), it is thought that a combination of several physical mechanisms could explain the high $\mathcal{M} / \mathcal{L}$ ratios of DSCs: $i$ ) systemic rotation, $i i)$ significant dark matter (DM) content, and/or iii) central massive BHs, however, the validity of these explanations has not been tested in numerical simulations.

First, we look at rotation in globular clusters. Some Milky Way GCs show slow rotation with amplitudes of several km $\mathrm{s}^{-1}$ (e.g. Bianchini et al. 2013, Kacharov et al. 2014), which

bovill@stsci.edu are at least one order of magnitude slower than what would be required to fully explain the $\mathcal{M} / \mathcal{L}$ ratios of DSCs. In fact, the required rotational velocities would destabilize the DSCs, and likely unbind the systems (T15). With that in mind, DSC rotation cannot be entirely ruled out, but rotation and tri-axiality cannot entirely account for their elevated $\mathcal{M} / \mathcal{L}$ ratios.

We next discuss the possible influence of dark matter on globular cluster formation and evolution. It has been proposed that Milky Way GCs may have formed within DM halos (Peebles 1984; Bromm \& Clarke 2002). If so, they must have lost the majority of the dark matter via dynamical processes throughout their evolution, since today there is no evidence for significant DM content in GCs (e.g. Moore 1996; Baumgardt et al. 2009, Lane et al. 2010; Conroy et al. 2011; Feng et al. 2012; Ibata et al. 2013; Hurst et al. 2015). For a discussion of MOND-based explanations, see Derakhshani (2014), however, MOND predictions fall orders of magnitude below what is required to explain the derive DSC dynamics (Smith $\&$ Candlish, private communication).

Finally, we discuss the evidence for and implications of central IMBHs in GCs. Observational limitations and biases (e.g. van den Bosch \& de Zeeuw 2010; Bianchini et al. 2015) have prevented the confirmation of central intermediate-mass BHs (IMBHs) in Local Group GCs (e.g. Sun et al. 2013; Kamann et al. 2014; Lützgendorf et al. 2015, Cseh et al. 2015. Wrobel et al. 2015). For example, the Milky Way GC M10 does not seem to possess the cuspy central surface density profile indicative of a central black hole, but the presence of an IMBH with a mass up to $0.75 \%$ of the total GC mass, corresponding to $\mathcal{M}_{\bullet} \approx 600 M_{\odot}$, cannot be excluded (Umbreit \& Rasio 2013). In any case, $\mathrm{BH}$ signatures in more massive CSSs have been definitively detected (Gebhardt et al. 2005. van der Marel \& Anderson 2010; Seth et al. 2014). A central cluster of BHs would not necessarily produce the cuspy observational signature of an IMBH (Banerjee \& Kroupa 2011), 
however such a dark cluster would be unable to duplicate the extremely high central densities derived for the DSCs.

The satellite system of Centaurus A within $\sim 50 \mathrm{kpc}$ of galactocentric distance is dominated by its rich known GC system, with over 600 confirmed members (van den Bergh et al. 1981; Hesser et al. 1984, 1986, Harris et al. 1992; Peng et al. 2004, Woodley et al. 2005, 2010; Rejkuba et al. 2007; Beasley et al. 2008), many of which populate the highluminosity (ie. high mass) end of the globular cluster luminosity function (GCLF). The total expected population may be as high as 2000 (Harris et al. 1984, 2002, 2010, 2012), but the distribution at large galacto-centric radii is relatively unconstrained. At these large radii, the known satellites of Centaurus A are limited to a few dozen confirmed dwarf galaxies within $\sim 1.4 \mathrm{Mpc}$ of galactocentric distance (e.g. Côté et al. 1997; Banks et al. 1999; Jerjen et al. 2000; Karachentsev et al. 2002, 2007; Crnojevic et al. 2014, Tully et al. 2015), but this population may not be complete due to the small sky coverage and shallow surface brightness limits of previous photometric surveys.

In this work, we present results from high-resolution $\Lambda \mathrm{CDM}$ numerical simulations of a Centaurus $\mathrm{A}$ analog halo. Based on our results we put upper limits on the central DM densities in the putative DSC candidates, and, by inference, provide stringent direct estimates of potential central $\mathrm{BH}$ masses. This paper is laid out as follows: Our high-resolution simulations of the Centaurus A analog halo are described in $\$ 2$, and the constraints provided by the known observed properties of the DSCs are given in $\$ 3$ We highlight the relevant circular velocity and density profiles of the dark matter halos compared to the measured stellar velocity dispersions of the DSCs in $\$ 4$, and the possibilities for the formation and growth of a central massive black hole in these systems in $\$ 5$. Finally, observational tests for our model are provided in $\$ 5.1$ and our summary and conclusions are presented in $\$ 6$

\section{SIMULATIONS}

In this section, we describe a suite of high resolution cosmological simulations of an isolated Centaurus A analog from $z=150$ to $z=0$. All initial conditions were generated with MUSIC (Hahn \& Abel 2011) and simulations were run with GADGET 2 (Springel 2005). Halo properties were analyzed using Amiga (Gill et al. 2004, Knollmann \& Knebe 2009) and merger trees generated with CONSISTENT_TREES (Behroozi et al. 2013). Code testing and preliminary simulations were performed on the GERYON cluster at the Institute of Astrophysics at Pontificia Universidad Católica de Chile, and all subsequent high-resolution simulations were run on the University of Maryland HPCC DEEPTHOUght 2.

We initially ran a $100 \mathrm{Mpc} / \mathrm{h}$ box with $N=256^{3}$, resolving Centaurus A candidates at $z=0$ with $>1000$ particles. All simulations are run from $z=150$ to $z=0$ with outputs every 10 Myr for $z>6$ and every 100 Myr for $z<6$. Our Centaurus A analog halo is chosen to have a total virial mass of $\sim 10^{13} M_{\odot}$, corresponding to the upper range of virial masses measured for Centaurus A (van den Bergh 2000; Peng et al. 2004, Woodley et al. 2007, 2010, Łokas 2008; Harris et al. 2014). Our Centaurus A analog is also roughly consistent with, if slightly more centrally concentrated than, measurements of $v_{\max } \sim 100 \mathrm{~km} \mathrm{~s}^{-1}$ at $7-22 \mathrm{kpc}$ (Israel 1998) and $\sim 3 \times 10^{11} M_{\odot}$ within $\sim 50 \mathrm{kpc}$ (Mathieu et al. 1996). To ap- proximate the Local Volume, we use an isolation criterion of no halos with $\mathcal{M}_{\text {vir }}>10^{12} M_{\odot}$ within $3 \mathrm{Mpc} / \mathrm{h}$ of the Centaurus $\mathrm{A}$ analog at $z=0$. We do not account for the presence of M83, which is $\sim 1 \mathrm{Mpc}$ away from Centaurus A; however, this should not bias our results significantly, as M83 is $\sim 2-3 R_{\text {vir }}$ away from Centaurus A. As we focus on the inner virial halo of Centaurus $A(<350 \mathrm{kpc})$ regions of Centaurus $A$, thus the presence (or lack) of an M83 analog will not change the properties and distribution of subhalos within our region of interest. This assumption closely matches those adopted while running an isolated Milky Way analog without an M31 counterpart to study Galactic satellite populations (e.g. Bullock \& Johnston 2005; Johnston et al. 2008, Springel et al. 2008; Diemand et al. 2007).

Once we identify our Centaurus A analog, we resimulate the Lagrangian region corresponding to $\sim 4 R_{\mathrm{vir}}(\sim 2.4 \mathrm{Mpc})$ at $z=0$ at higher resolution to produce a clean region of $r \simeq 2 R_{\mathrm{vir}}$ at $z=0$. We perform two resimulations, at $N_{\text {eff }}=4096^{3}$ with $m_{p}=1.13 \times 10^{6} M_{\odot}$ and $\epsilon=500$ pc physical (run 100M_4096) and at $N_{\mathrm{eff}}=8192^{3}$ with $m_{p}=1.4 \times 10^{5} M_{\odot}$ and $\epsilon=200 \mathrm{pc}$ physical (100M_8192). These resolutions were chosen to resolve halos with $M>10^{8} M_{\odot}$ with 100 and 1000 particles respectively. $N_{\text {eff }}$ is the number of particles in the simulation if the entire $100 \mathrm{Mpc} / \mathrm{h}$ box were simulated at the highest resolution and $\epsilon$ is the gravitational softening length. Our two re-simulations resolve halos with masses above $1.13 \times 10^{8} M_{\odot}$ and $1.4 \times 10^{7} M_{\odot}$, respectively, with 100 particles. Table 1 provides a summary of these simulations and the properties of the respective Centaurus A analogs. All halo parameters are those derived by AміgA for the Centaurus A analog at $z=0$. $R_{v i r}$ of a given halo depends on both $M_{v i r}$ and $v_{\text {max }}$, but in a non-linear way.

Before studying the satellite population of our Centaurus A analog halo in detail, we ensure our simulations are numerically convergent. We plot the velocity function of the Centaurus $\mathrm{A}$ sub-halos for both re-simulations in Figure 1 and find our simulation to be convergent to the resolution limit. While the low-resolution run (100M_4096) resolves halos down to circular velocities of $v_{\max } \approx 10 \mathrm{~km} \mathrm{~s}^{-1}$, in our higher-resolution run (100M_8192) we resolve approximately 7000 subhalos to $v_{\max } \approx 5 \mathrm{~km} \mathrm{~s}^{-1}$.

\section{CONSTRAINTS FROM THE DARK STAR CLUSTERS}

\subsection{General Considerations}

As stated in $\$ 1$, we are determining whether the DSCs can be explained by a past or present subset of dark matter subhalos around Centaurus A. The next step in our analysis is to determine what, if any, constraints the measured baryonic properties of these systems can place on the dark matter halo characteristics.

Under the assumption that the observed stellar velocity dispersion values are unbiased measurements of the stellar velocity dispersion ${ }^{1}$. we estimate the central mass density for each DSC that is not accounted for by the baryonic component. In the framework of our model, we investigate whether the DSCs can be explained by the presence of past and/or present-day dark matter. Note, in our analysis, we consider only two mass components, the observed stellar mass and a "dark" compo-

\footnotetext{
${ }^{1}$ For a detailed discussion on statistical and systematic uncertainties of the velocity dispersion measurements, see Taylor et al. (2015).
} 
TABLE 1

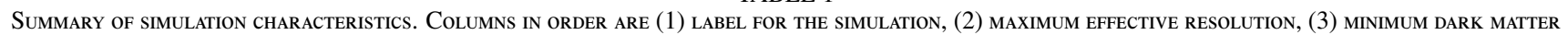
PARTICLE MASS, (4) PHYSICAL SOFTENING LENGTH IN PC/H, (5) NUMBER OF PARTICLES IN THE CENTAURUS A halo, (6) Virial MASS OF THE CENTAURUS A halo IN UNITS OF $10^{13} M_{\odot}$, (2) maximum circular velocity of the Centaurus A halo in KM s ${ }^{-1}$, and (7) Virial radius of the Centaurus A halo in Kpc/h.

\begin{tabular}{cccccccc}
\hline \hline Run & $N_{\text {eff }}$ & $\begin{array}{c}m_{p} \\
\left(M_{\odot}\right)\end{array}$ & $\begin{array}{c}\epsilon \\
(\mathrm{pc})\end{array}$ & $N_{\text {part }}$ & $\begin{array}{c}M_{\text {Centaurus A }} \\
\left(10^{13} M_{\odot}\right)\end{array}$ & $\begin{array}{c}v_{\max }^{\text {Centaurus A }} \\
\left(\mathrm{km} \mathrm{s}^{-1}\right)\end{array}$ & $\begin{array}{c}R_{\mathrm{vir}} \\
\left(\mathrm{kpc} \mathrm{h}^{-1}\right)\end{array}$ \\
\hline 100M_4096 & $4096^{3}$ & $1.2 \times 10^{6}$ & 500 & 10334710 & 1.16444 & 437.33 & 368.60 \\
100M_8192 & $8192^{3}$ & $1.4 \times 10^{5}$ & 200 & 83263765 & 1.17269 & 437.78 & 369.46 \\
\hline \hline
\end{tabular}

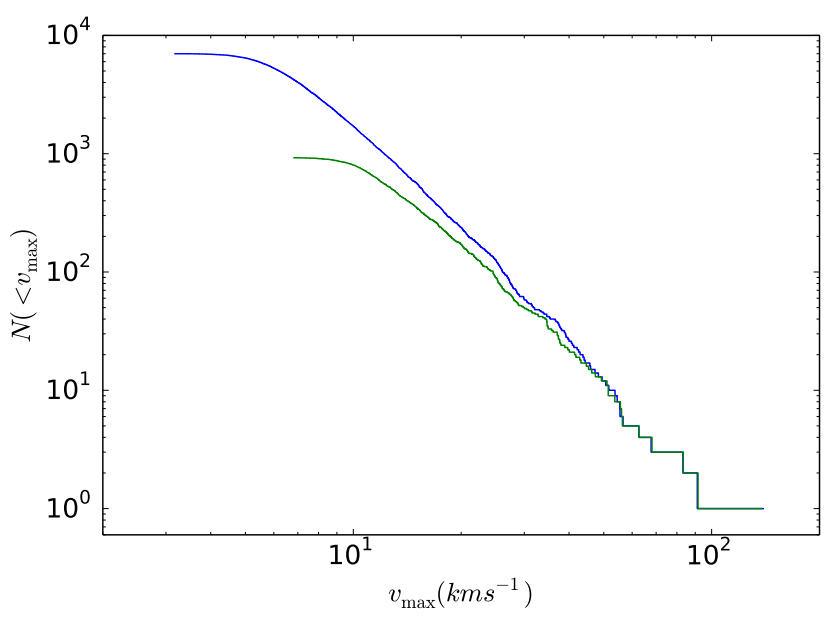

FIG. 1.- Velocity function of the Centaurus A sub-halos at $z=0$ for the 100M_8192 (blue curve) and 100M_4096 run (green curve).

nent which that we presume to be $i$ ) a central cusp of dark matter and/or ii) a massive central black hole.

\subsection{Observational Constraints}

The main constraint for our model comes from the observed stellar properties of the DSCs. We know that, at $z=0$, they have $>10^{5} M_{\odot}$ of stars with high central stellar densities. GCs and ultra-faint dwarfs (Brown et al.2014) are among the oldest stellar systems in the universe. It has been proposed that a fraction of the ultra-faint dwarfs are fossils of the first galaxies (Bovill \& Ricotti 2009, 2011a b). In this work we explore whether the DSCs could be a new category of ancient stellar systems which also formed before reionization, but with a much higher stellar density than the ultra-faint dwarfs. We assume that, like, the Milky Way GCs, they formed the majority of their stars $>12$ Gyr ago (Katz \& Ricotti 2013). During this epoch of the first galaxies, the majority of stars formed in dense, globular cluster like, environments (Calvi et al. 2014 Ricotti 2002; Katz \& Ricotti 2013).

If the local star formation efficiency within the cluster is $f_{\star}^{\text {local }} \gtrsim 0.35$ and/or the cluster forms $N_{\star}>10^{6}$ stars, the cluster will survive the expulsion of its initial gas reservoir and remain bound for a Hubble time (e.g. Parmentier \& Fritze 2009; Lamers et al. 2010, Elmegreen 2014). Given $\mathcal{M}_{z=0} / \mathcal{M}_{\text {initial }}=9.1$ (Prieto \& Gnedin 2008), at least $\sim 10 \%$ of the $z=0$ population of the DSCs formed in an initial, dense burst at high redshift.

Assuming these systems form stars with a stellar IMF with a characteristic mass of $1 M_{\odot}$, this requires there to be at least $10^{5.5} M_{\odot}$ of gas in the inner $\sim 20 \mathrm{pc}$ of the dark matter halo. The $\sim 20$ pc scale is chosen to corresopnd to the physical scale of the DSCs at $z=0$. Note, that this is a lower estimate since we can expect a large fraction of the stars to be stripped when the tidal radius reaches the stellar population, after the accretion into a more massive halo (e.g. Peñarrubia et al. 2008, Smith et al. 2013, 2015).

While we do not assume that DSCs formed all of their stars at high redshift, we do assume, in contrast with the progenitors of the ultra faint dwarfs, that their initial burst of star formation was highly concentrated. This is in line with recent work on the formation of the first galaxies which suggests that while many of the first galaxies formed diffuse stellar systems (Ricotti et al. 2002a b, 2008), a subset formed denser stellar populations (Pawlik et al. 2011, 2013).

\subsection{Dark Matter Halo Framework}

The need for $\sim 10^{5}-10^{6} M_{\odot}$ of gas in the halo core gives us an estimate for the approximate dark matter mass within $20 \mathrm{pc}$ required in the DSC progenitors at early times. Assuming the initial gas fraction of the halos follows the cosmic baryon fraction, $f_{b}$, we can estimate the dark matter mass in the core, $\mathcal{M}_{\mathrm{DM} \text {,core }} \approx \mathcal{M}_{g} / f_{b}$. From this required $\mathcal{M}_{\mathrm{DM} \text {,core }}$, we approximate the required virialization redshift, $z_{\mathrm{vir}}$, and its mass at virialization, $\mathcal{M}_{\text {vir }}\left(z_{\text {vir }}\right)$. Assuming the halos approximate an NFW profile (Navarro et al. 1996) at virialization:

$$
\rho_{\mathrm{NFW}}(r)=\rho_{o}\left\{\frac{r}{R_{s}}\left(1+\frac{r}{R_{s}}\right)^{2}\right\}^{-1}
$$

where $R_{s}$ is the scale radius of the halo and $\rho_{o}$ the central density. $\rho_{\mathrm{NFW}}$ can be written solely as a function of $z_{\mathrm{vir}}$ and $\mathcal{M}_{\text {vir }}\left(z_{\text {vir }}\right)$

$$
\rho_{\mathrm{NFW}}\left\{r, z_{\mathrm{vir}}, \mathcal{M}_{\mathrm{vir}}\left(z_{\mathrm{vir}}\right)\right\}
$$

since

$$
3 \cdot 10^{3} \times \Omega_{M}\left(1+z_{\mathrm{vir}}\right)^{3}=\Delta_{c}\left(\frac{c^{3}}{\ln (1+c)+\frac{c}{1+c}}\right)
$$

where $\mathrm{c}$ is the concentration and $\Delta_{c}$ is the overdensity required for halo collapse as a function of the halo's concentration, and

$$
R_{\mathrm{vir}}=1.5 \mathrm{kpc}\left(\frac{\Omega_{M} h^{2}}{0.147}\right)^{-1 / 3}\left(\frac{\mathcal{M}_{\mathrm{vir}}}{10^{8} M_{\odot}}\right)^{1 / 3}\left(\frac{10}{1+z_{\mathrm{vir}}}\right) .
$$

If we assume a spherically symmetric dark matter distribution, we obtain the dark matter core mass:

$$
\mathcal{M}_{\mathrm{DM}, \text { core }}=4 \pi \rho_{o} R_{s}^{3}\left[\ln \left(\frac{R_{s}+R_{\text {core }}}{R_{s}}\right)-\frac{R_{\text {core }}}{R_{s}+R_{\text {core }}}\right]
$$

where $R_{\text {core }}$ is the radius of the core, here assumed to be $20 \mathrm{pc}$. 


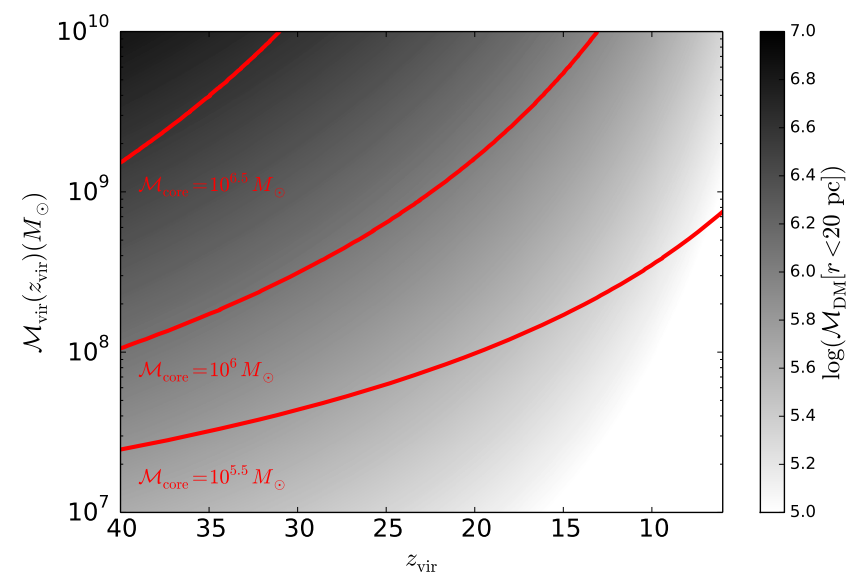

FIG. 2.- Contour plot of the mass of dark matter within $20 \mathrm{pc}$ for a given $z_{\mathrm{vir}}$ and $\mathcal{M}_{\mathrm{vir}}\left(z_{\mathrm{vir}}\right)$. The range of masses on the greyscale run from $10^{5} M_{\odot}$ to $10^{7} M_{\odot}$ straddling our approximate value for the core dark matter mass required to form a DSC progenitor of $10^{6} M_{\odot}$.

Using Equation 5, we plot the contours of $\mathcal{M}_{\mathrm{DM} \text {,core }}$ as a function of $z_{\text {vir }}$ and $\mathcal{M}_{\text {vir }}\left(z_{\text {vir }}\right)$ in Figure 2, where we show contours of equivalent $\mathcal{M}_{\mathrm{DM}}(r<20 \mathrm{pc})$ in greyscale. We also plot the $\mathcal{M}_{\text {vir }}\left(z_{\text {vir }}\right)$ curves for $\mathcal{M}_{\mathrm{DM}}(r<20 \mathrm{pc})=$ $\left[10^{5.5} M_{\odot}, 10^{6} M_{\odot}, 10^{6.5} M_{\odot}\right]$. Note that dark matter halos with $\mathcal{M}_{\mathrm{DM}}(r<20 \mathrm{pc}) \simeq 10^{6} M_{\odot}$ would form $\sim 10^{5.5} M_{\odot}$ of stars at $f_{\star}^{\text {local }}=0.35$, making them plausible progenitors of the DSCs. From these curves we estimate $\mathcal{M}_{\text {vir }}\left(z=z_{\text {vir }}\right)>10^{8} M_{\odot}$, which is in agreement with the masses required for the formation of $\sim 10^{6} M_{\odot}$ proto-GC systems (Katz \& Ricotti 2013)

From the $\mathcal{M}_{\text {vir }}\left(z_{\text {vir }}\right)$ required to produce the necessary $\mathcal{M}_{\mathrm{DM} \text {,core }}$ and given the growth of halos before infall into Centaurus A, we assume any possible progenitor of a DSC reached a peak mass $\mathcal{M}_{\text {vir }}\left(z_{\text {peak }}\right)>10^{9} M_{\odot}$ before falling into Centaurus $\mathrm{A}$, where $\mathcal{M}_{\text {vir }}\left(z_{\text {peak }}\right)$ is the maximum virial mass of a halo.

The $z=0$ mass function of all resolved Centaurus A subhalos in 100M_8192 is shown in the left panel of Figure 3 in red, with the mass function of the subset of subhalos with $\mathcal{M}_{\text {vir }}\left(z_{\text {peak }}\right)>10^{9} M_{\odot}$ overlaid in black. These latter halos are considered potential progenitors of the DSCs found by T15.

The number of $z=0$ subhalos with $M_{\text {vir }}\left(z_{\text {peak }}\right)>M_{\text {threshold }}$ is shown in the right panel of Figure 3. We plot both the total number of subahlos, $N_{\text {subhalos }}$ with $M_{\text {vir }}\left(z_{\text {peak }}\right)>M_{\text {threshold }}$ in the Centaurus A analog's virial halo and $N_{\text {subhalos }}$ for a given $M_{\text {threshold }}$ within $100 \mathrm{kpc}$ projected into the plane of the sky. The projected distance of $100 \mathrm{kpc}$ is chosen because all the DSCs are within $100 \mathrm{kpc}$ of Centaurus A (T15). We find 20 $z=0$ subahlos with $M_{\text {vir }}\left(z_{\text {peak }}\right)>10^{9} M_{\odot}$ within $100 \mathrm{kpc}$ projected of Centaurus A and 200 - 300 within the entire virial halo. Please note two caveats to these numbers. First, we do not include subhalos which are not detected by Amiga at $z=0$. Such halos are assumed to be completely destroyed and we have no way of determining their positions relative to the central galaxies at $z=0$. Secondly, in the outer regions of the halo, the $z=0$ subhalos must not only account for a potential additional population of DSCs, but the known dwarf satellites of Centaurus A (Karachentsev et al. 2007; van den Bergh 2000; Crnojevic̀ et al. 2014; Crnojević et al. 2016) as well.
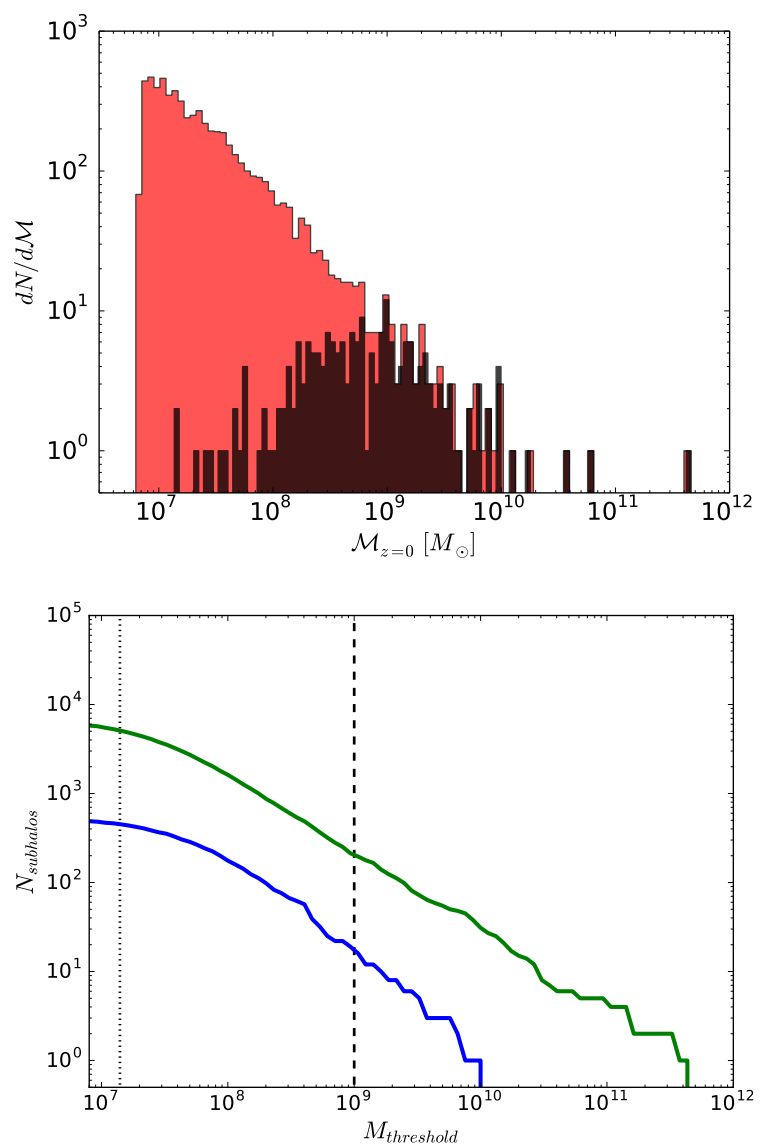

FIG. 3.- Left: Mass function of all the resolved subhalos at $z=0$ (red) and the subhalos with $\mathcal{M}_{\text {vir }}\left(z_{\text {peak }}\right)>10^{9} M_{\odot}$ (black), which are considered to be progenitor halos of DSCs discovered in Centaurus A. Right: Number of subhalos, $N_{\text {subhalos }}$ with a $M_{\text {vir }}\left(z_{\text {peak }}\right)>M_{\text {threshold }}$ in the virial halo of our Centaurus A analog (green) and $N_{\text {subhalos }}$ within $100 \mathrm{kpc}$, projected, of the central halo (blue) for a range of $M_{\text {threshold }}$. The mass of halos resolved with 100 particles in $100 M_{-} 8192$ is shown as the dotted line and the $M_{\text {vir }}\left(z_{\text {peak }}\right)>$ $10^{9} M_{\odot}$ determined from observational constraints is the dashed line.

\section{DARK MATTER HALO PROFILES AND CENTRAL DENSITIES}

We have established that the likely DSC progenitors have $\mathcal{M}_{\text {vir }}\left(z_{\text {peak }}\right)>10^{9} M_{\odot}$. However the DSCs have extremely high derived central densities, which naturally raises the question: do the Centaurus A subhalos with $\mathcal{M}_{\text {vir }}\left(z_{\text {peak }}\right)>10^{9} M_{\odot}$ have such high central densities at $z=0$ ?

\subsubsection{Profile Properties at various Redshifts}

Our simulations do not have the resolution to directly measure the subhalo densities in the inner $20 \mathrm{pc}$. We therefore fit a halo profile to the subhalos at various redshifts and extrapolate an estimate of the density from the simulation convergence radius at $\sim 600 \mathrm{kpc} / \mathrm{h}$ to $20 \mathrm{pc}$. We present a detailed discussion of the convergence of our profiles in Appendix A. Since NFW profiles are not accurate fits for subhalos (Stoehr 2006), we use the Einasto profiles (Einasto 1965; Navarro et al. 2004) to fit subhalo profiles at $z=0$. The Einasto profile approximates the radial dark matter density profile with the following analytical form:

$$
\rho(r)=\rho_{-2} e^{\frac{-2}{\alpha}\left[\left(\frac{r}{r_{-2}}\right)^{\alpha}-1.0\right]}
$$


where $r_{-2}$ is the radius at which the slope is $-2, \rho_{-2}$ is the density at that point and $\alpha$ is between 0.16 and 0.20 (Springel et al. 2008). For $\alpha=0.18$ this becomes,

$$
\rho(r)=99.5 M_{\odot} p c^{-3} \frac{v_{\max }}{r_{\max }} e^{\frac{-2}{\alpha}\left[\left(\frac{2.189 x}{r_{\max }}\right)^{\alpha}-1.0\right],}
$$

where $r_{\max }$ and $v_{\max }$ are halo parameters derived by Amiga. In Figure 4, we plot the Einasto density profiles fit to the subhalos with $\mathcal{M}_{\text {vir }}\left(z_{\text {peak }}\right)>10^{9} M_{\odot}$ and compare them to the derived densities for the various star cluster populations in Centaurus A (see T15). The Einasto profiles are shaded according to their $z=0$ dark matter mass. We find that the even the densest subhalos in our simulation at $z=0$ have central densities more than an order of magnitude too low to account for the average DSC.

We also show the Stoehr profiles (Stoehr et al. 2002) to fit subhalo profiles at $z=0$. The Stoehr profile approximates the circular velocity using:

$$
\log \left(\frac{v_{c}}{v_{\max }}\right)=-a\left(\log \left\{\frac{r}{r_{\max }}\right\}\right)^{2},
$$

where $a$ is a parameter fit to the resolved portions of the circular velocity curve calculated by AmIGA. In Figure 4, we plot the circular velocity curves of the Stoehr profile fits for the subhalos with $\mathcal{M}_{\text {vir }}\left(z_{\text {peak }}\right)>10^{9} M_{\odot}$ and compare to the lineof-sight radial velocities for the various star cluster populations in Centaurus A (see T15). The Stoehr profiles are shaded according to their $z=0$ dark matter mass. Although some Stoehr profiles with the highest central densities match some of the lowest-density DSCs, we find that, as with the Einasto profiles, the subhalos in our simulation at $z=0$ have central densities more than an order of magnitude too low to account for the average DSC. As such, the inability of dark matter alone to account for the high derived densities of the DSC is independent of our choice of subhalo profile.

However, we are not only interested in the dark matter density at $z=0$, but also in the central density of halos at $z_{\text {peak }}$, when these were still actively forming stars, before their evolution became dominated by Centaurus A. In the left panel of Figure 5, we plot the NFW profiles of the halos at $z_{\text {peak }}$. In both panels of Figure 5, we use the same symbol colors as for the data points in Figure 4, however, the NFW profiles in both panels are shaded by $\mathcal{M}_{\text {vir }}\left(z_{\text {peak }}\right)$ rather than $\mathcal{M}_{\mathrm{DM}}(z=0)$.

Before stating categorically that dark matter alone cannot account for the densities of a significant fraction of the DSCs, we check one final set of high-redshift subhalos. The progenitors of densest star clusters in Centaurus A likely formed at high redshifts. As redshift increases so does the fraction of progenitors which do not survive as $z=0$ subhalos. However, the right panel of Figure 5 shows that, even at high redshift (e.g. $z=9.3$ ), there are no Centaurus A progenitors or $z=0$ subhalos which have dark matter central densities high enough to explain the average observed central densities of most observed star clusters from the T15 sample.

\subsubsection{Excess Central Densities}

The critical difference between the DSCs, UCDs and GCs is clear upon further study of Figures $4 \& \& 5$. For all these populations, we assume a stellar $\mathcal{M} / \mathcal{L}$ ratio $\left(\mathcal{M} / \mathcal{L}_{\star}\right)$ to estimate the stellar mass of each system. The extensively studied
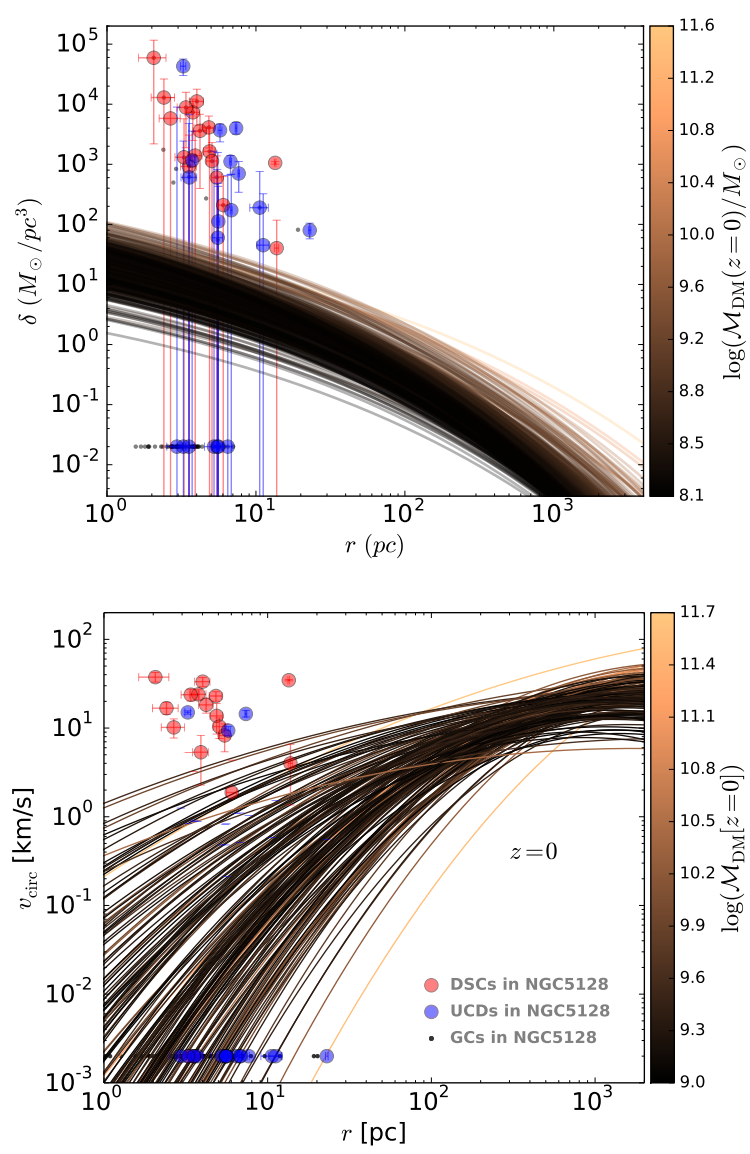

Fig. 4.-Left: Einasto halo profiles for the $z=0$ subhalos of Centaurus A with $\mathcal{M}_{\text {vir }}\left(z_{\text {peak }}\right)>10^{9} M_{\odot}$ with the derived central densities for the DSCs (red symbols), UCDs (blue symbols), and GCs (black dots). The density profile colour of each halo is parametrized by the halo mass at $z=0$ $\left(\mathcal{M}_{\mathrm{DM}}(z=0)\right)$ as indicated by the colour scale on the right side of the panel. Right: Stoehr profiles for the subhalos of the Centaurus A analog with the $\sigma_{r_{1 / 2}}$ versus $r_{h l}$ of the DSCs, UCDs and GCs of Centaurus AThe Stoehr profiles and DSCs, UCDs and GCs have the same colors as symbols as for the Einastro profiles.

Milky Way GC system has a typical $\mathcal{M} / \mathcal{L}_{\star} \simeq 2.2$. Comprehensive studies of the $\mathcal{M} / \mathcal{L}_{\star}$ values in the Centaurus A system currently do not exist. We therefore estimate the typical $\mathcal{M} / \mathcal{L}_{\star}$ for the Centaurus A GC system by measuring the peak of the $\mathcal{M}_{\mathrm{dyn}} / \mathcal{L}$ distribution for the GCs (black points), and approximate $\mathcal{M} / \mathcal{L}_{\star} \sim 4$.0. In this idealized scenario, we assume the mass of the Centaurus A GCs is entirely accounted for by their stellar populations.

In the following, each density excess measure is derived using

$$
\rho_{\text {excess }}=\rho_{\text {dyn }}-\rho_{\text {baryon }}=\frac{\mathcal{M}_{\mathrm{dyn}}-\mathcal{L}\left(\mathcal{M} / \mathcal{L}_{\star}\right)}{\frac{4}{3} \pi r_{e}^{3}}
$$

where $\mathcal{M}_{\mathrm{dyn}}$ is computed from the observed $\sigma_{\star}, \mathcal{L}$ is the derived V-band luminosity assuming a distance modulus, $m-$ $M=27.88$ (Harris et al. 2010), $r_{e}$ is the effective radius and $\mathcal{M} / \mathcal{L}_{\star}$ is the stellar mass-to-light ratio in the V-band. For visualization purposes, if an object has $\rho_{\text {excess }}<0$ we assign an arbitrary $\rho_{\text {excess }}=2 \times 10^{-2}$. Such objects are not unphysical but have $\mathcal{M} / \mathcal{L}_{\star}<4$.0. With a reasonable $\mathcal{M} / \mathcal{L}_{\star}$, the dynam- 

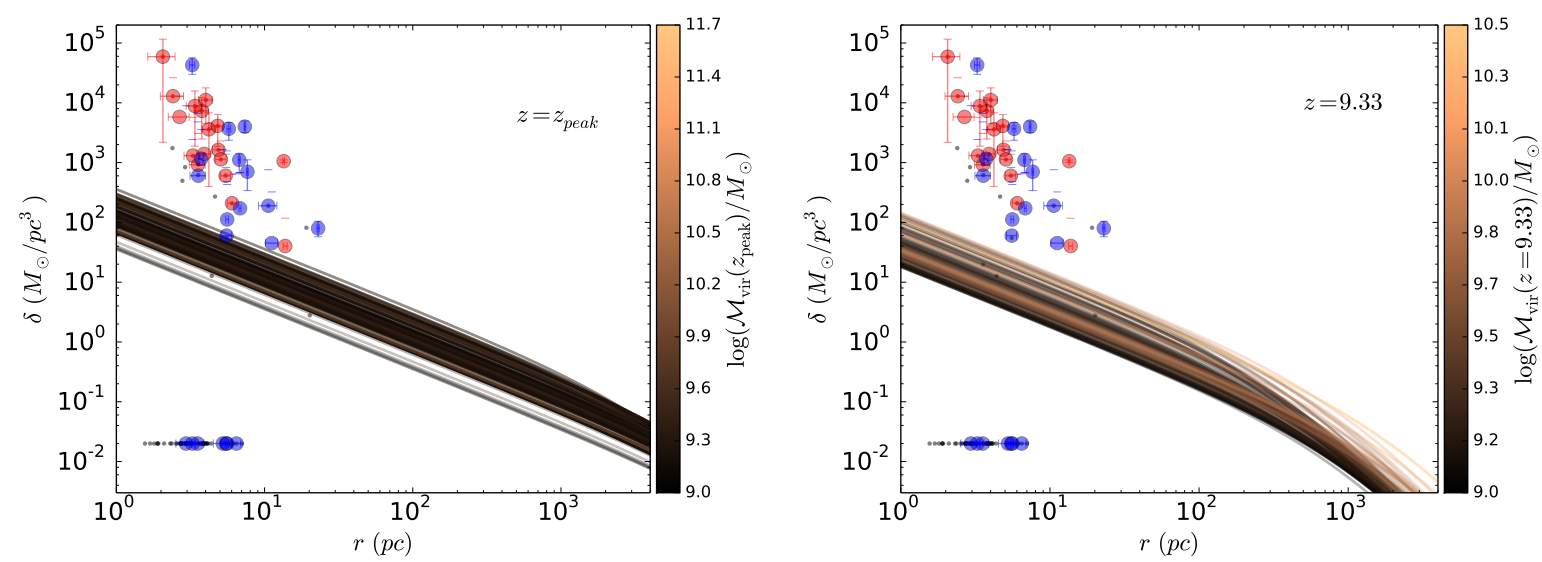

FIG. 5.- (Left panel): NFW profile fits based on the AMIGA parameters derived at $z_{\text {peak }}$ for each $z=0$ Centaurus A subhalos with $\mathcal{M}_{\text {vir }}\left(z_{\text {peak }}\right)>10^{9} M_{\odot}$ with the derived central density of the DSCs at $z=0$. (Right panel): NFW profiles of the Centaurus A progenitors at $z=9.3$. The NFW profiles are color coded with $\mathcal{M}_{\text {vir }}(z)$ at the corresponding redshifts. The central densities derived for the Centaurus A star cluster population are as in Figure 4

ical mass of a significant fraction of the GCs and UCDs can be accounted for with their stars alone. This is not the case for the DSCs. While there is evidence that stellar populations in elliptical galaxies and UCDs have bottom heavy IMFs, for both UCDs and ellipticals the bottom heavy IMFs increase the stellar $M / L$ by no more than a factor of five (Cappellari et al. 2012, Mieske \& Kroupa 2008; Dabringhausen et al. 2008). This is not enough to account for the measured dynamical $M / L$ s of the DSCs $(10-100)$.

We find that dark matter alone cannot account for the high observed central densities $\left(\rho_{\text {excess }} \gtrsim \rho_{\text {DM }}\right.$, see Figures $4 \& 5$. Assuming the observations are correct, the only physical possibility to explain $10^{5}-10^{7} M_{\odot}$ of mass within $\sim 10 \mathrm{pc}$ radius is a central black hole in the SMBH range. T15 assumed that the DSCs fall on the $\mathcal{M}_{\bullet}-\sigma$ relation. However, the validity of this assumption for tidally stripped systems is questionable. In the subsequent analysis, we will not make this assumption, but rather derive the required $\mathcal{M}_{\text {• to account }}$ for the missing central mass density of our subhalos $\left(\rho_{\text {excess }}\right)$ that cannot be accounted for by the DM density profiles and the stellar mass distribution. We estimate the central blackhole mass of the clusters with either $\mathcal{M}_{\bullet}=\mathcal{M}_{\text {dyn }}-\mathcal{M}_{\star}-\mathcal{M}_{\mathrm{DM}}$ or $\mathcal{M}_{\bullet}=\mathcal{M}_{\text {dyn }}-\mathcal{M}_{\star}$. In the former, we assume a cusp of the original dark matter halo has survived and, in the latter, we assume a purely baryonic stellar system at $z=0$. The difference is negligible as $\mathcal{M}_{\mathrm{DM}}(r<10 \mathrm{pc}) \ll \mathcal{M}_{\mathrm{dyn}}$ for even the densest halos.

We find the central black hole must have a mass in the range $\mathcal{M}_{\bullet} \approx 10^{5}-10^{7} M_{\odot}$, well above the estimates for IMBH candidates in GCs (e.g. Lützgendorf et al.2013), however this is not beyond the range seen in dwarf galaxies (Reines \& Volonteri 2015). Therefore, the embedding of DSC progenitors in DM halos remains a critical component of their formation and evolution.

\section{THE CENTRAL BLACK HOLE}

We established in Section 4 that dark matter alone cannot reproduce the observed densities of the DSCs, and only a black hole in the $10^{5}-10^{7} M_{\odot}$ mass range can account for the observed dynamics. We illustrate in Figure 6 the distributions of the derived black hole masses for UCDs and DSCs. Therefore, a critical component of our model is the formation and growth of a central black hole which reaches at least $\mathcal{M}_{\bullet}>10^{5} M_{\odot}$ before the infall of the host halo into Centaurus A.

In the following, we assume for simplicity that the central black hole seed grows only via gas accretion at a constant accretion rate over cosmic time. We define an accretion ratio, $\lambda / \epsilon$, which is the quotient between the Eddington ratio $(\lambda)$ over the mass-energy conversion efficiency $(\epsilon)$. The Eddington ratio, $\lambda=\dot{\mathcal{M}}_{\text {acc }} / \dot{\mathcal{M}}_{\text {Edd }}$ is the fraction of the Eddington accretion rate sustained by the central black hole. To get a physical accretion rate in $M_{\odot} /$ yr from $\lambda$ we need to assume an efficiency for the conversion of mass to energy, $\epsilon$, as the matter falls onto the central black hole. Since the Eddington limit is set by the luminosity, lower $\epsilon$ will allow more mass to be accreted onto the black hole for the same $\lambda$.

While the required $z=0$ black hole masses are large for GCs (see Figure 6), they are on the low end of the SMBH range. We will initially look at Bondi accretion onto these systems. The Bondi accretion rate depends on the black hole mass and the density of the surrounding gas. For an IMBH, $\lambda_{\text {Bondi }}=\dot{\mathcal{M}}_{\text {Bondi }} / \dot{\mathcal{M}}_{\text {Edd }} \approx 10^{-4}$ (Kong et al. 2010) and radiative feedback from an IMBH gives a true accretion rate of $1 \%$ of Bondi (Park \& Ricotti 2011) giving a $\lambda \simeq 10^{-6}$. We assume $\epsilon=0.1$, which is reasonable for thin disk accretion if we ignore black hole spin (Shapiro 1973; Park \& Ostriker 2001). This gives us an accretion ratio of approximately $\lambda / \epsilon \simeq 10^{-5}$.

However, with Bondi accretion it is not possible to grow the central black hole to the necessary mass before infall into Centaurus A unless the seed black hole is above $10^{5} M_{\odot}$. We therefore assume the black holes are accreting well above the Bondi rate, consistent with coevolution of a $\mathrm{BH}$ and bulge (Park et al. 2015).

Figure 6 illustrates the mass growth of three different seed black holes with masses $1000 M_{\odot}, 300 M_{\odot}$ and $10 M_{\odot}$, with the assumption that the seed formed concurrently with the first generation of Pop-II stars. The $1000 M_{\odot}$ black hole seed approximates the remnant of a runaway stellar collision (black curves). The $300 M_{\odot}$ seed (dark orange curves) is the lower bound of the range of masses required for direct collapse (Heger et al.2003). The final set of light orange curves is for a $10 M_{\odot}$ black hole seed, which approximates the growth via 

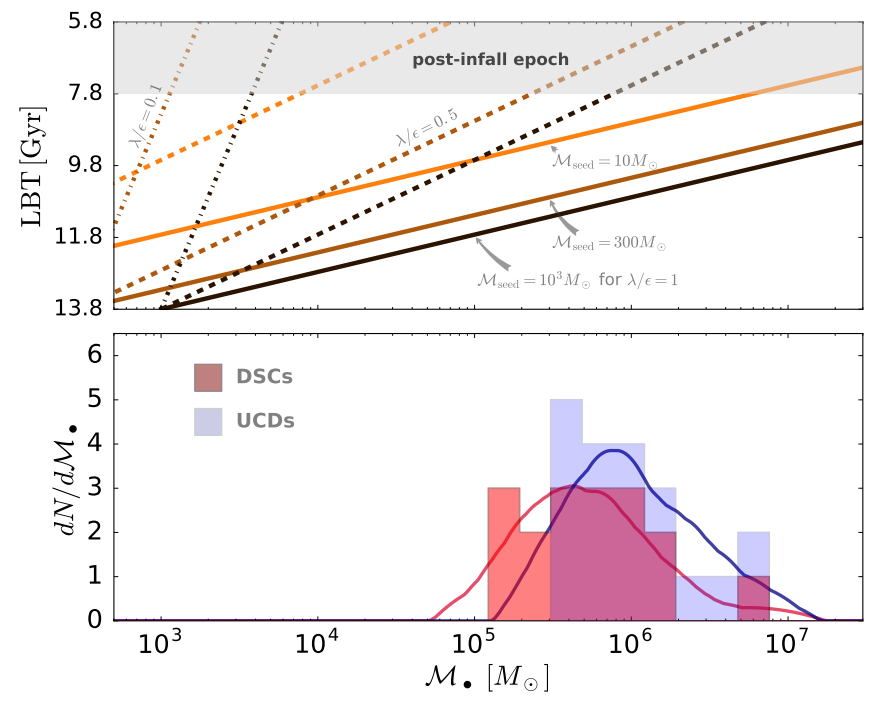

FIG. 6.- (Top panel): The growth of three different seed black hole masses for a variety of $\lambda / \epsilon$. We choose $\mathcal{M}_{B H}=10 M_{\odot}$ (light orange) to represent a CCSN remnant, $\mathcal{M}_{B H}=300 M_{\odot}$ (dark orange) and $\mathcal{M}_{B H}=10^{3} M_{\odot}$ (black) to approximate the remnant of a runaway stellar collision. We explore three different values of $\lambda / \epsilon=[1,0.5,0.1]$ as the solid, dashed, and dotted lines, respectively. For thin disk accretion this corresponds to $10 \%, 5 \%$ and $1 \%$ of Eddington accretion. (Bottom panel): Distributions of the central black hole masses necessary to explain the excess dynamical mass that cannot be explained by dark matter densities, derived for the high- $\mathcal{M} / \mathcal{L}$ stellar systems in Centaurus A, i.e. UCDs and DSCs (see T15). The curves indicate Epanechnikov kernel non-parametric probability density estimates.

accretion onto a core-collapse SN remnant. Further study is needed to determine if our toy model for black hole accretion holds up to a more physically rigorous treatment.

To estimate the required accretion ratio to reach the $10^{5}-$ $10^{7} M_{\odot}$ black-hole mass range derived from observations (see histograms in Figure 6), we use two criteria. First, the gas supply to the central black hole will be extinguished by ram pressure stripping after the DSC progenitor falls into Centaurus A. Second, we know that by $z=0$ the DSC has lost the vast majority of its dark matter and stars, suggesting the near complete destruction of the DSC progenitor halo. Therefore, the seed black hole must reach the observed mass range (i.e. $>10^{5} M_{\odot}$ ) by $z \approx 1$ in order to allow its host halo enough time to be destroyed via interactions with Centaurus A during the post-infall epoch. It is clear from Figure 6 that, as long as $\lambda / \epsilon \gtrsim 1$, it is quite possible to grow the measured black hole masses from a $300 M_{\odot}$ seed and even from a $10 M_{\odot}$ seed by $z \approx 1$, i.e. before the post-infall epoch begins and the environment of the black hole is evacuated by ram-pressure stripping and other interactions in the host halo.

\subsection{Observational Tests for the Model}

We propose that the DSCs, stripped of their dark matter and outer stars, are remnant stellar cusps surrounding central IMBH/SMBHs. Our model for the formation of the DSCs is roughly as follows.

1. During the epoch of the first galaxies a dense, massive $\left(M>10^{5} M_{\odot}\right)$ central cluster forms in a dark matter halo.

2. Rapid segregation of the most massive stars to the core results in a runaway stellar collision (Portegies Zwart et al. 2004, van der Marel 2004). This collision produces a seed black hole of several hundred to several thousand solar masses (Katz et al. 2015).

3. Over the next several billion years the halo continues to accrete gas, feeding additional star formation and the central black hole. During this era, feedback from bursty star formation precludes the formation of a significant bulge (Guedes et al.2011, Governato et al. 2010).

4. Between 5-10 Gyr ago, the dwarf falls into Centaurus A . This allows enough time for its gas, dark matter and outer stars are completely stripped (Smith et al. 2013, 2015), therefore the DSCs would not be hosted in the surviving subhalos at $z=0$. At $z=0$, the only remaining sign of the lost dwarf is the remnant cusp of stars surrounding its central SMBH. Excepting its dynamics and metallicity, the surviving stellar cusp resembles a GC.

Since these systems resemble GCs photometrically and structurally, it is only possible to disentangle their origin as lost dwarf galaxies through the dynamic signatures of their central black holes and the following observational criteria.

Chemical Composition: If the DSCs formed as dwarf galaxies their residual stellar populations should accordingly have dwarf galaxy-like chemical abundances; namely, a significantly elevated metallicity $[\mathrm{Fe} / \mathrm{H}]>-2$ dex, a measurable metallicity spread $\left(\sigma_{[\mathrm{Fe} / \mathrm{H}]}>0\right)$, and a higher $[\alpha / \mathrm{Fe}]$ ratio than expected for their present stellar mass on the $[\alpha / \mathrm{Fe}]-$ luminosity relation of stellar systems (e.g. Thomas et al.2005, Smith et al. 2009). In addition, they should be above expectations from the $\mathrm{L}-[\mathrm{Fe} / \mathrm{H}]$ for $\mathcal{M}_{\star}=10^{5}-10^{6} M_{\odot}$ systems. From the selective core-collapse $\mathrm{SN}$ enrichment we would also expect enhanced light-element (CNO) to iron ratios, as well as a significant variance in iron-peak elements.

X-ray flux: While the central black hole is likely now quiescent, low-level accretion $\left(\sim 10^{-8}\right.$ Eddington $)$ is possible via AGB winds and planetary nebulae. At Centaurus A's distance, even these low accretion rates would be detectable by Chandra or XMM for the expected central black hole masses.

\section{SUMMARY AND CONCLUSIONS}

We have used a new set of high resolution simulations of an isolated $10^{13} M_{\odot}$ Centaurus A analog to investigate possible formation models for the DSCs found in T15. We assume the DSCs formed within dark matter halos before determining which Centaurus A subhalos at $z=0$ have the highest probability of being DSC progenitors.

We find that DSCs likely formed in dark matter halos which virialized at high redshift with $\mathcal{M}_{\text {vir }}\left(z_{v i r}\right)>10^{8} M_{\odot}$. Since dark matter halos grow in mass in isolation, we assume that any DSC progenitor had $\mathcal{M}_{\text {vir }}\left(z_{\text {peak }}\right)>10^{9} M_{\odot}$. However, no dark matter profile associated with the Centaurus A system, whether for the subhalos at $z=0, z_{\text {peak }}$, or high redshift (eg. $z=9.3$ ), has a high enough central density to account for the dynamical mass derived for the DSCs. Even the densest DM halos fall one to two orders of magnitude short of explaining the DSCs as a population. Therefore, we find that dark matter 
alone cannot account for the currently measured dynamical masses in the DSC cores.

The only class of objects that can account for $>10^{5} M_{\odot}$ of mass within $10 \mathrm{pc}$ of the cluster center are massive central black holes. We find that the dynamical masses of the DSCs can be accounted for by central black holes between $10^{5}-10^{7} M_{\odot}$. While evidence for IMBHs has been found in several globular clusters, the mass range required to explain the DSCs is several orders of magnitude higher. However, such supermassive black holes would need to form in a galaxy. Therefore, while dark matter cannot explain the $z=0$ dynamics of the DSCs, the derived central black hole masses strongly support their formation and early evolution within dark matter halos.

Future work is required to constrain the number and distribution of these objects around Centaurus A and to determine if there are likely to be any lost dwarfs masquerading as glob- ular clusters in our own Milky Way.

\section{ACKNOWLEDGMENTS}

We wish to thank Nelson Padilla, Chris Reynolds and John Wise for useful discussions and Derek Richardson for help with the University of Maryland HPCC. MSB acknowledges the support of FONDECYT Project Grant (No. 3130549). MAT acknowledges the financial support provided by an excellence grant from the Vicerrectora de Investigacin and the Institute of Astrophysics Graduate School Fund at Pontificia Universidad Catlica de Chile and the European Southern Observatory Graduate Student Fellowship program. This research was supported by FONDECYT Regular Grant (No. 1161817) and BASAL Center for Astrophysics and Associated Technologies (PFB-06). The authors acknowledge the University of Maryland supercomputing resources (http://www.it.umd.edu/hpcc) made available for conducting the research reported in this paper.

\section{REFERENCES}

Banks, G. D., Disney, M. J., Knezek, P. M., et al. 1999, ApJ, 524, 612

Banerjee, S., \& Kroupa, P. 2011, ApJL, 741, L12

Baumgardt, H., Côté, P., Hilker, M., et al. 2009, MNRAS, 396, 2051

Beasley, M.A., Bridges, T., Peng, E., et al. 2008, MNRAS, 386, 1443

Behroozi, P. S., Wechsler, R. H., Wu, H.-Y., et al. 2013, ApJ, 763, 18

Bianchini, P., Varri, A. L., Bertin, G., \& Zocchi, A. 2013, ApJ, 772, 67

Bianchini, P., Norris, M. A., van de Ven, G., \& Schinnerer, E. 2015, MNRAS, 453, 365

Bovill, M. S., \& Ricotti, M. 2011, ApJ, 741, 17

Bovill, M. S., \& Ricotti, M. 2011, ApJ, 741, 18

Bovill, M. S., \& Ricotti, M. 2009, ApJ, 693, 1859

Bromm, V., \& Clarke, C. J. 2002, ApJL, 566, L1

Brown, T. M., Tumlinson, J., Geha, M., et al. 2014, ApJ, 796, 91

Bullock, J. S., \& Johnston, K. V. 2005, ApJ, 635, 931

Calvi, V., Stiavelli, M., Bradley, L., Pizzella, A., \& Kim, S. 2014, ApJ, 796, 102

Cappellari, M., McDermid, R. M., Alatalo, K., et al. 2012, Nature, 484, 485

Conroy, C., Loeb, A., \& Spergel, D. N. 2011, ApJ, 741, 72

Côté, S., Freeman, K. C. , Carigan, C., \& Quinn, P. J. 1997, AJ, 114, 1313

Crnojevic̀, D., Sand, D. J., Caldwell, N., et al. 2014, ApJL, 795, L35

Crnojević, D., Sand, D. J., Spekkens, K., et al. 2016, ApJ, 823, 19

Cseh, D., Webb, N. A., Godet, O., et al. 2015, MNRAS, 446, 3268

Dabringhausen, J., Hilker, M., \& Kroupa, P. 2008, MNRAS, 386, 864

Derakhshani, K. 2014, ApJ, 783, 48

Di Cintio, A., Brook, C. B., Macciò, A. V., et al. 2014, MNRAS, 437, 415

Diemand, J., Kuhlen, M., \& Madau, P. 2007, ApJ, 657, 262

Elmegreen, B. G. 2014, arXiv:1410.1075

Einasto, J. 1965, Trudy Astrofizicheskogo Instituta Alma-Ata, 5, 87

Feng, L., Yuan, Q., Yin, P.-F., Bi, X.-J., \& Li, M. 2012, Journal of

Cosmology and Astroparticle Physics, 4, 030

Gao, L., Navarro, J. F., Frenk, C. S., et al. 2012, MNRAS, 425, 2169

Gebhardt, K., Rich, R. M., \& Ho, L. C. 2005, ApJ, 634, 1093

Gill, S. P. D., Knebe, A., \& Gibson, B. K. 2004, MNRAS, 351, 399

Governato, F., Brook, C., Mayer, L., et al. 2010, Nature, 463, 203

Governato, F., Zolotov, A., Pontzen, A., et al. 2012, MNRAS, 422, 1231

Guedes, J., Callegari, S., Madau, P., \& Mayer, L. 2011, ApJ, 742, 76

Hahn, O., \& Abel, T. 2011, MNRAS, 415, 2101

Han, J., Cole, S., Frenk, C. S., \& Jing, Y. 2016, MNRAS, 457, 1208

Harris, G. L. H., Hesser, J. E., Harris, H. C. \& Curry, P. J. 1984, ApJ, 287, 175

Harris, G. L. H., Geisler, D., Harris, H. C., \& Hesser, J.E. 1992, AJ, 104, 613

Harris, G. L. H., Geisler, D., Harris, W. E., \& Hesser, J. E. 2002b, in IAU

Symp. 207, Extragalactic Star Clusters, ed. D. P. Geisler, E. K. Grebel, \& D. Minniti (San Francisco, CA: ASP), 309

Harris, G. L. H., Rejkuba, M., \& Harris, W. E. 2010, Publications of the Astronomical Society of Australia, 27, 457

Harris, G. L. H., Gómez, M., Harris, W. E., et al. 2012, AJ, 143, 84

Harris, G. L. H., Poole, G. B., \& Harris, W. E. 2014, MNRAS, 438, 2117
Heger, A., Fryer, C. L., Woosley, S. E., Langer, N., \& Hartmann, D. H. 2003, ApJ, 591, 288

Hesser, J. E., Harris, H. C., van den Bergh, S., \& Harris, G. L. H. 1984, AJ, 276,491

Hesser, J. E., Harris, H. C., \& Harris, G. L. H. 1986, ApJ, 303, L51

Hurst, T. J., Zentner, A. R., Natarajan, A., \& Badenes, C. 2015, Physical Review D, 91, 103514

Ibata, R., Nipoti, C., Sollima, A., et al. 2013, MNRAS, 428, 3648

Israel, F. P. 1998, A\&A Rev., 8, 237

Jerjen, H., Freeman, K. C., \& Binggeli, B. 2000, AJ, 119, 166

Johnston, K. V., Bullock, J. S., Sharma, S., et al. 2008, ApJ, 689, 936

Kacharov, N., Bianchini, P., Koch, A., et al. 2014, A\&A, 567, A69

Kamann, S., Wisotzki, L., Roth, M. M., et al. 2014, A\&A, 566, A58

Karachentsev, I. D., Sharina, M. E., Dolphin, A. E., et al. 2002, A\&A, 385,

21

Karachentsev, I. D., Tully, R. B., Dolphin, A., et al. 2007, AJ, 133, 504

Katz, H., \& Ricotti, M. 2013, MNRAS, 432, 3250

Katz, H., Sijacki, D., \& Haehnelt, M. G. 2015, MNRAS, 451, 2352

Knollmann, S. R., \& Knebe, A. 2009, ApJs, 182, 608

Kong, A. K. H., Heinke, C. O., di Stefano, R., et al. 2010, MNRAS, 407, L84

Lane, R. R., Kiss, L. L., Lewis, G. F., et al. 2010, MNRAS, 406, 2732

Lamers, H. J. G. L. M., Baumgardt, H., \& Gieles, M. 2010, MNRAS, 409, 305

Łokas, E. L. 2008, ApJL, 680, L101

Lützgendorf, N., Kissler-Patig, M., Neumayer, N., et al. 2013, A\&A, 555, A26

Lützgendorf, N., Gebhardt, K., Baumgardt, H., et al. 2015, A\&A, 581, A1

Madau, P., Shen, S., \& Governato, F. 2014, ApJL, 789, L17

Mashchenko, S., Couchman, H. M. P., \& Wadsley, J. 2006, Nature, 442, 539

Mashchenko, S., Wadsley, J., \& Couchman, H. M. P. 2008, Science, 319, 174

Mathieu, A., Dejonghe, H., \& Hui, X. 1996, A\&A, 309, 30

Mieske, S., \& Kroupa, P. 2008, ApJ, 677, 276-282

Moore, B. 1996, ApJL, 461, L13

Navarro, J. F., Frenk, C. S., \& White, S. D. M. 1996, ApJ, 462, 563

Navarro, J. F., Hayashi, E., Power, C., et al. 2004, MNRAS, 349, 1039

Norris, M. A., Escudero, C. G., Faifer, F. R., et al. 2015, MNRAS, 451, 3615

Park, M.-G., \& Ostriker, J. P. 2001, ApJ, 549, 100

Park, K., \& Ricotti, M. 2011, ApJ, 739, 2

Park, K., Ricotti, M., Natarajan, P., Bogdanović, T., \& Wise, J. H. 2015, arXiv: 1512.03434

Parmentier, G., \& Fritze, U. 2009, ApJ, 690, 1112

Pawlik, A. H., Milosavljević, M., \& Bromm, V. 2011, ApJ, 731, 54

Pawlik, A. H., Milosavljević, M., \& Bromm, V. 2013, ApJ, 767, 59

Peebles, P. J. E. 1984, ApJ, 277, 470

Peñarrubia, J., Navarro, J. F., \& McConnachie, A. W. 2008, ApJ, 673, 226

Peng, E. W., Ford, H. C., \& Freeman, K. C. 2004, ApJ, 602, 685

Pontzen, A., \& Governato, F. 2012, MNRAS, 421, 3464

Portegies Zwart, S. F., Baumgardt, H., Hut, P., Makino, J., \& McMillan,

S. L. W. 2004, Nature, 428,724 
Prieto, J. L., \& Gnedin, O. Y. 2008, ApJ, 689, 919

Read, J. I., \& Gilmore, G. 2005, MNRAS, 356, 107

Reines, A. E., \& Volonteri, M. 2015, ApJ, 813, 82

Rejkuba, M., Dubath, P., Minniti, D., \& Meylan, G. 2007, A\&A, 469, 147

Renaud, F., Bournaud, F., Emsellem, E., et al. 2013, MNRAS, 436, 1836

Renaud, F., Bournaud, F., \& Duc, P.-A. 2015, MNRAS, 446, 2038

Ricotti, M. 2002, MNRAS, 336, L33

Ricotti, M., Gnedin, N. Y., \& Shull, J. M. 2002a, ApJ, 575, 33

Ricotti, M., Gnedin, N. Y., \& Shull, J. M. 2002b, ApJ, 575, 49

Ricotti, M., Gnedin, N. Y., \& Shull, J. M. 2008, ApJ, 685, 21

Rodriguez-Puebla, A., Behroozi, P., Primack, J., et al. 2016, arXiv: 1602.04813

Shapiro, S. L. 1973, ApJ, 180, 531

Seth, A. C., van den Bosch, R., Mieske, S., et al. 2014, Nature, 513, 398

Smith, R. J., Lucey, J. R., Hudson, M. J., \& Bridges, T. J. 2009, MNRAS, 398, 119

Smith, R., Sánchez-Janssen, R., Fellhauer, M., et al. 2013, MNRAS, 429, 1066

Smith, R., Sánchez-Janssen, R., Beasley, M. A., et al. 2015, arXiv: 1509.02537

Sun, M.-Y., Jin, Y.-L., Gu, W.-M., et al. 2013, ApJ, 776, 118

Springel, V. 2005, MNRAS, 364, 1105

Springel, V., Wang, J., Vogelsberger, M., et al. 2008, MNRAS, 391, 1685

Stoehr, F., White, S. D. M., Tormen, G., \& Springel, V. 2002, MNRAS, 335,

L84
Stoehr, F. 2006, MNRAS, 365, 147

Taylor, M. A., Puzia, T. H., Gomez, M., Woodley, K. A., 2015, ApJ, 805, 65

Teyssier, R., Pontzen, A., Dubois, Y., \& Read, J. I. 2013, MNRAS, 429, 3068

Thomas, D., Maraston, C., Bender, R., \& Mendes de Oliveira, C. 2005, ApJ, 621,673

Tully, R. B., Libeskind, N. I., Karachentsev, I. D., et al. 2015, ApJL, 802, L25

Umbreit, S., \& Rasio, F. A. 2013, ApJ, 768, 26

van den Bergh, S., Hesser, J. E., \& Harris, G. L. H 1981, AJ, 86, 24

van den Bergh, S. 2000, AJ, 119, 609

van den Bosch, R. C. E., \& de Zeeuw, P. T. 2010, MNRAS, 401, 1770

van der Marel, R. P. 2004, Coevolution of Black Holes and Galaxies, 37

van der Marel, R. P., \& Anderson, J. 2010, ApJ, 710, 1063

Woodley, K. A., Harris, W. E., \& Harris, G. L. H. 2005, AJ, 129, 2654

Woodley, K. A., Harris, W. E., Beasley, M. A., et al. 2007, AJ, 134, 494

Woodley, K. A., Gómez, M., Harris, W. E., Geisler, D., \& Harris, G. L. H. 2010, AJ, 139, 1871

Wrobel, J. M., Nyland, K. E., \& Miller-Jones, J. C. A. 2015, arXiv:1507.07613

APPENDIX

\section{CONVERGENCE TESTS}

With half light radii of less than $20 \mathrm{pc}$, comparing the derived DSC densities to the dark matter halo profiles in our simulations requires us to extrapolate two orders of magnitude from the convergence radius of our simulations $(3 \epsilon \sim 600 \mathrm{pc}$ physical). We chose not to run simulations with a convergence radius of a few parsecs because of the computational expense and the increasing importance of baryonic effects of the dark matter distribution on sub-kiloparsec scales (Read \& Gilmore 2005; Mashchenko et al. 2006, 2008; Governato et al. 2010, 2012; Teyssier et al.|2013; Di Cintio et al. 2014;, Pontzen \& Governato 2012; Madau et al. 2014).

It is standard practice to use derived dark matter density profiles to study the densities at or the softening length of the simulation (Han et al. 2016; Rodriguez-Puebla et al. 2016, Gao et al. 2012). We use information derived from AmIGA to fit a dark matter density profile and extrapolate that profile into the inner $20 \mathrm{pc}$ of the halo and determine the densities of the inner dark matter profiles of our DSC candidate progenitors at various redshifts.

To test the convergence of our halo density profiles and therefore our estimates of the dark matter density within $20 \mathrm{pc}$, we compare the density profile fits for our DSC progenitors in the 100M_8192 and 100M_4096 runs. The properties of these runs are summarized in Table 1 in the main text.

We remind the reader that the DSC candidates are chosen to be resolved by Amiga at $z=0$ with $M_{\text {vir }}\left(z_{\text {peak }}\right)>10^{9} M_{\odot}$. We only investigate those $z=0$ subhalos which are resolved with $N>100$ particles, though AmIGA is robust to $N>50$ (Knollmann \& Knebe 2009). Throughout the analysis in this appendix the curves and points for the 100M_4096 will be shown in green and the curves and points for the 100M_8192 will be shown in black.

For the $z=0$ density profiles we use the Einasto profile (Einasto 1965; Navarro et al. 2004) with an assumed $\alpha=0.18$ (Springel et al. 2008). For a chosen $\alpha$, the Einasto profile thus reduces to a relatively simple function dependent on $r_{\max }$ and $v_{\max }$. We first choose the $N_{4096}$ subhalos in $100 \mathrm{M}_{-} 4096$ which have $M_{\text {vir }}\left(z_{\text {peak }}\right)>10^{9} M_{\odot}$ are resolved with $N>100$ at $z=0$. To check the convergence between the two simulations, we select the $N_{4096}$ most massive $z=0$ subhalos in 100M_8192 with $M_{\text {vir }}\left(z_{\text {peak }}\right)>10^{9} M_{\odot}$ and compare the $v_{\max }, r_{\max }$ and $r_{\text {vir }}$ distributions for 100M_4096 versus 100M_8192 (Figure 77. For all three parameters, we find good agreement between the distributions in 100M_4096 and 100M_8192. We next explicitly look at the densities of the extrapolated Einasto profiles at $10 \mathrm{pc}$. In Figure 8 we show the distribution of the extrapolated $\rho(r=10 p c)$ for 100M_4096 versus 100M_8192 (left panel) and the distributions of $\rho(r=10 p c)$ for both runs (right panel). While there is not a one to one match between the halos, we find their distributions to be the same. In addition the density range inhabited by the DSCs (red shading) cannot be accounted for by the extrapolated dark matter densities in either simulation.

We therefore state the distributions of our $z=0$ Einasto profiles are convergent and any differences between individual halos does not change our result that the extremely high derived densities of the DSCs cannot be account for by dark matter alone.

We next determine the convergence of our NFW density profiles at $z_{\text {peak }}$. Since the NFW profile can be expressed as $\rho_{N F W}\left(r, c_{v i r}, R_{v i r}\right)$, we first confirm that the DSC candidate progenitors in 100M_4096 and 100M_8192 have the same distribution in $c_{v i r}$ and $R_{v i r}$ (see Figure 9 before showing that they produce the same distribution of dark matter densities to $10 \mathrm{pc}$ (right panel of Fig 10). For both 100M_4096 and 100M_8192 the halos are well resolved at $z_{\text {peak }}$ with $N>1000$ and $N>10^{4}$ respectively.

We find the distribution of $c_{v i r}$ versus $R_{v i r}$ are extremely similar for 100M_4096 and 100M_8192. As with the Einasto profiles at $z=0$, we follow up the tests for convergence of the halo parameters with a confirmation that the distributions of the extrapolated 

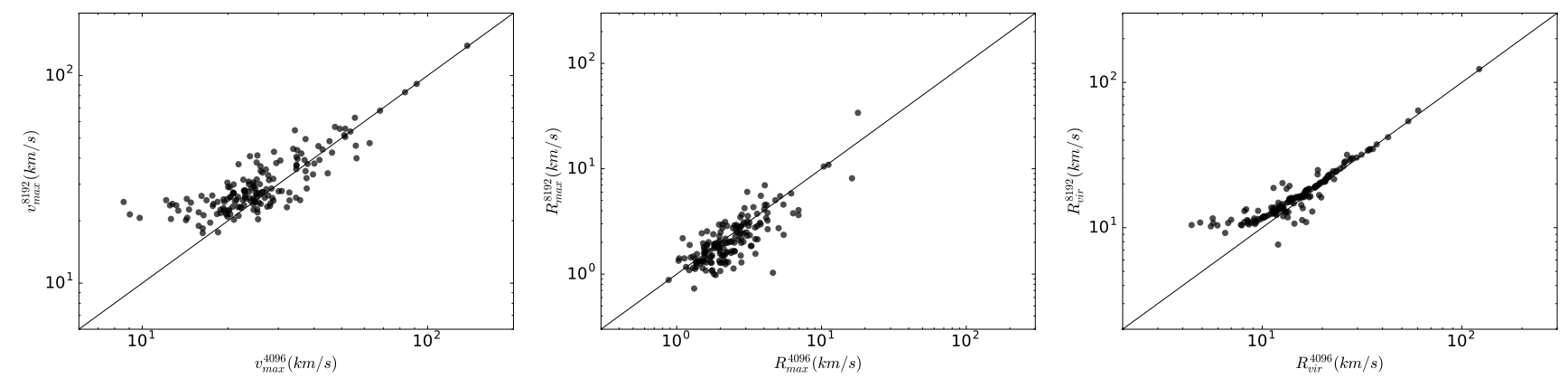

FIG. 7.- For the DSC candidate progenitor subhalos resolved in 100M_4096 and their counterparts in 100M_8192 we plot $v_{\max }$ (left panel), $r_{\max }$ (center panel), and $r_{v i r}$ (right panel). In each panel, the thin black line shows equivalence.
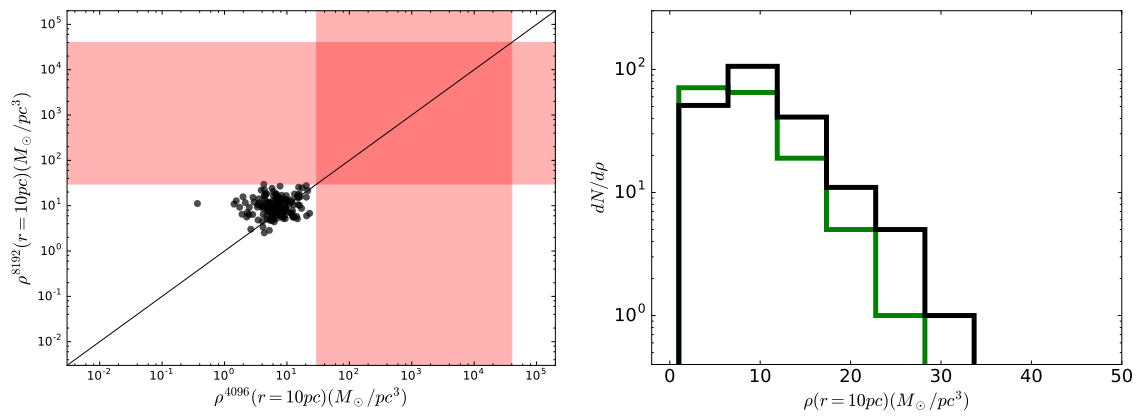

FIg. 8. - Left: The extrapolated Einasto densities for the DSC candidates at $z=0$ for the 100M_8192 versus 100M_4096 (black points). The distribution of the derived densities for the DSCs are shown as the red shaded area. Right: Histogram of the distributions of the $z=0$ densities at extrapolated to $10 \mathrm{pc}$ using the Einasto profile for 100M_4096 (green) and 100M_8192 (black).

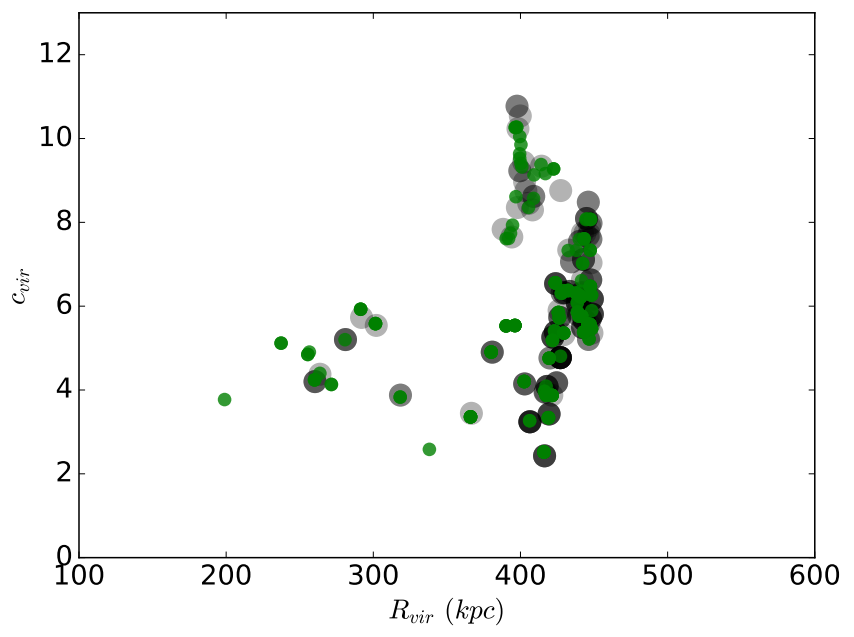

FIG. 9.- Distribution of $c_{N F W}\left(z_{\text {peak }}\right)$ versus $R_{\text {vir }}\left(z_{\text {peak }}\right)$ for 100M_4096 (green circles) and 100M_8192 (black circles).

densities at $10 \mathrm{pc}$ are equivalent (see right panel of Figure 10 As with the $z=0$ Einasto profiles, we do not have a perfect one to one matching of our extrapolated densities, however the results from 100M_4096 and 100M_8192 are once again equilvalent. The dark matter densities at $z_{\text {peak }}$ were also at least an order of magnitude too low to account for the derived central densities of the DSCs.

As we show good agreement between 100M_4096 and 100M_8192 for both the $z=0$ Einasto profiles and the $z_{\text {peak }}$ NFW profiles in both their properties and extrapolated densities, we hold our extrapolation form the convergence radius of $600 \mathrm{pc}$ to within $20 \mathrm{pc}$ to be valid and robust for our analysis. 

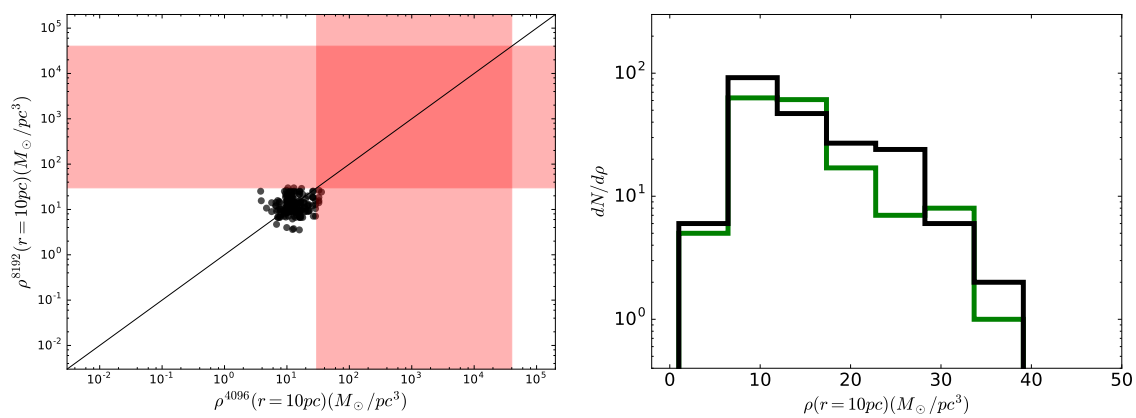

Fig. 10._Left: The extrapolated NFW densities for the DSC candidates at $z=z_{\text {peak }}$ for the 100M_8192 versus 100M_4096 (black points). The distribution of the derived densities for the DSCs are shown as the red shaded area. Right: Histogram of the distributions of the $z=z_{\text {peak }}$ densities at extrapolated to 10 pc using the NFW profile for 100M_4096 (green) and 100M_8192 (black). 